\title{
Design and Evaluation of a Virtual Gearshift Application
}

\author{
M. Tideman, M. C. van der Voort, and F. J. A. M. van Houten
}

\begin{abstract}
When a customer buys a new car, he or she wants it to address personal preferences with respect to its driving behavior. By utilizing Virtual Reality technology, a Virtual Prototyping Environment (VPE) can be created in which the behavior of a vehicle or part of a vehicle can be evaluated and adjusted to match the driver's desires. This paper describes the design and the evaluation of a VPE for manually operated gearboxes. The test group considered the simulated "virtual" gearshift feel to be quite similar to the "real" gearshift feel of a test vehicle. By further developing this VPE, it should become possible to define gearshift feel by customer assessment through haptic simulation, after which the physical gearbox is designed in such a way that it matches the preferred shifting behavior.
\end{abstract}

\section{INTRODUCTION}

W HEN buying a new passenger car, the customer not only demands the vehicle to be reliable, safe, comfortable and attractive, but in addition wants it to address his or her personal preferences with respect to its driving behavior. Although one can commonly select different types of engines and transmissions per vehicle type, it is not possible to customize these engines and transmissions any further. Reasons lay in production and mechanical limitations as well as in the fact that people have difficulty to quantify their wishes as it comes to describing the desired "feel" of a vehicle or part of a vehicle. A good example is the feeling experienced while operating a gearbox. People are very well able to express the feel of the gearbox in terms of stiff, loose, smooth, clunky, etc. However, if they would prefer the gearshift feel to be altered, they are unable to express their desires. For instance, if they would prefer the gearbox to feel more stiff, they are unable to tell how much stiffer it should become. This makes it very hard for designers to address

Manuscript received December 12, 2003.

M. Tideman is with the Laboratory of Design, Production and Management of the University of Twente, P.O. Box 217, 7500 AE Enschede, The Netherlands (phone: +31-53-489-3192; fax: +31-53-4893631; e-mail: m.tideman@utwente.nl).

M. C. van der Voort is with the Laboratory of Design, Production and Management of the University of Twente, P.O. Box 217, 7500 AE Enschede, The Netherlands (e-mail: m.c.vandervoort@utwente.nl).

F. J. A. M. van Houten is with the Laboratory of Design, Production and Management of the University of Twente, P.O. Box 217, 7500 AE Enschede, The Netherlands (e-mail: f.j.a.m.vanhouten@utwente.nl). these customer wishes.

A solution to this problem would be if potential customers could easily compare several different gearboxes or several variants of the same gearbox. By identifying the preferences of target groups and thereby defining the desired gearshift feel, gearboxes could be designed in such a way that it matches the customer desires better. This process however requires an environment in which a great number of different gearboxes can be evaluated without actually building physical prototypes. A Virtual Prototyping Environment (VPE) is such an environment.

A VPE can be created by utilizing Virtual Reality interfaces (e.g. haptic devices, stereoscopic displays, 3D sound devices). VR interfaces record the user's actions and -in turn- stimulate his senses. In this way, the user gets the illusion of having some kind of interaction. A VPE enables evaluation of specific characteristics of a candidate design without having to build a physical prototype. When coupled to a CAD system, a VPE enables inverting the order in which the design process is gone through. Instead of creating geometry and evaluating the behavior afterwards, the product behavior can be defined after which the corresponding geometry is created [1]. This so-called behavior based design provides a tool for direct integration of customer wishes into the design, as they will be able to adjust the behavior of a future product so that it exactly meets their wishes. In this way, the design process is no longer necessarily the exclusive domain of the designer and as a result, his subjective way of interpretation and translation of customer wishes is locked out.

At the laboratory of Design, Production and Management of the University of Twente, research is performed on creation of environments for virtual prototyping applications. One of the projects is the development of a VPE for manual transmissions in passenger cars. This paper describes the design and evaluation of a Virtual Gearshift Application, i.e. an application that raises the illusion of manually shifting gears in a passenger car when operated. By further developing this application, it should become possible to define the desired gearshift feel, after which the physical gearbox is designed in such a way that it matches the desires. In this way, gearboxes can be designed or adjusted 
for groups of drivers (old/young, sporty/comfy).

\section{APPROACH}

Until now, there are no findings on whether and how the gearshift feel of a manual transmission in a passenger car can be simulated. In order to obtain knowledge and gain experience on simulating gearshift feel, this research is limited to the development and evaluation of an application that simulates the feel of one single existing gearbox. Therefore, the gearlever of a passenger car is equipped with a system that measures the gearlever's motion related to the forces induced on the operator's hand. Based on measurements performed with this system, an application is designed that simulates this particular gearshift feel, i.e. this gearbox-specific relation between motion and forces. This application incorporates a commercially available haptic device. In order to determine whether the virtual gearshift feel complies with the real gearshift feel, a usability test is performed. Within this test, a group of ten participants is asked to compare the "gearboxes" by means of a questionnaire.

\section{THE GEARSHIFT FEEL}

Operation of a manual transmission in a passenger car takes place by means of interaction between the operator's hand and the user-interface of the transmission, i.e. the gear knob. Moving the gear knob is done by muscle power, applied through the skeletal system and the contact area between the hand and the gear knob. Depending on the resistance force induced by the complete transmission system and applied on the hand through the contact area, the totality of gear knob and hand executes a certain movement. So, the interaction is nothing but a relation between motion and forces.

Although the interaction between operator and gear knob and the so-called "gearshift feel" are closely connected, there is a subtle difference. As described above, the interaction can be expressed by an objective set of discrete motion and force data. However, when this interaction is experienced by the operator, a subjective layer is added. The operator will interpret the relation between motion and forces after.which he judges the gearbox to be stiff, loose, smooth, silky, clunky, rubbery, etc. The actual gearshift feel consists of both the objective and subjective layer.

This leads us to an important issue in designing and evaluating virtual environments. During the design phase, the interaction between user and real world is tried to be copied precisely. However, the quality of a virtual environment is not determined by the degree of perfection to which this copy has been made. It is determined by the degree to which the user interprets the copied interaction correctly, i.e. the degree to which the user gets the same experience as he would have had in the real world.
Therefore, in order to evaluate a virtual environment, the "real" and "virtual" experiences should be measured and compared rather than the "real" and "virtual" interactions.

In connection with this issue, the design of the Virtual Gearshift Application will be based on the measured interaction, i.e. the relation between motion and forces, whereas the evaluation will be done by measuring and comparing the user's experience. An adequate way to measure experiences is by exploiting a questionnaire.

\section{DESIGN OF THE VIRTUAL GEARSHIFT APPLICATION}

\section{A. Measuring the Interaction}

The interaction is measured by a system mounted onto the test vehicle's gearlever. This system consists of a force sensor and a motion tracker. The system logs the gearlever's spatial orientation as well as the forces induced on the operator's hand. By taking the first and second time derivative of the gear knob's orientation, motion data (i.e. velocity and acceleration) can be extracted. Figure 1 shows the gearlever equipped with the measurement system as well as the original gearlever.

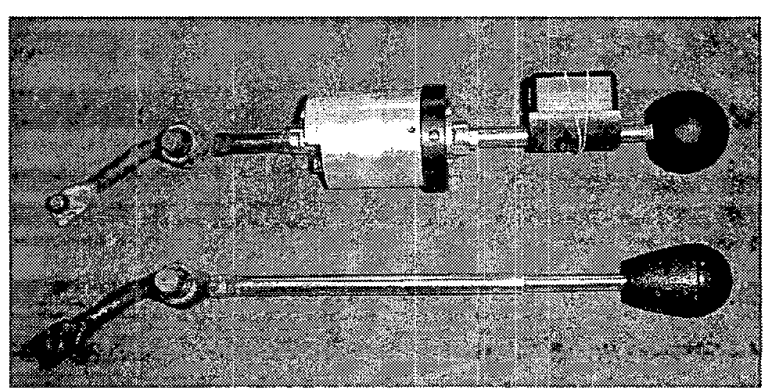

Fig. 1. The gearlever equipped with the measurement system (above) compared to the original gearlever (below).

Using this system, measurements are performed on the test vehicle's gearlever. Due to practical limitations, these measurements take place under "showroom-shifting" circumstances, i.e. while the vehicle is at rest, the engine is not running, and the clutch is disengaged. A group of ten participants is asked to operate the gearbox according to a number of prescribed patterns.

The main results of this testing are:

1) During gear shifting, the forces induced on the operator's hand do not show a significant variance for different operators;

2) During gear shifting, the forces induced on the operator's hand do not show any variance for different operating speeds;

3) During gear shifting, the forces induced on the operator's hand are mainly induced by the locking mechanism and the synchronization unit; 
4) As the locking mechanism of the gearbox consists of a spring-loaded ball that moves into a groove when a particular gear is engaged and out of it when a gear is disengaged, this effect may be modeled as compliance;

5) As the synchronization unit of the gearbox consists of two conical faces that make frictional contact just before a gear is engaged, this effect may be modeled as Coulomb friction.

\section{B. Selection of the Haptic Interface}

The interaction between operator and gear knob can be simulated by using a haptic interface. A haptic interface is a device configured to provide haptic information to a human. Just as a video interface allows the user to see a computer generated scene, a haptic interface permits the user to "feel" it [2].

Haptic interfaces have two basic functions [3]. The first is to measure forces, positions and their time-derivatives at the operator's hand (or other body locations). The second is to display forces and positions back to the operator under the control of the computer running the VR simulation.

There exist two fundamental methods for controlling haptic interfaces [4]. When position is input to the control loop and forces are fed back to the operator, we speak of impedance control. Alternatively, the simulation can use admittance control, in which forces applied to the end effector are sensed and positions are fed back through the haptic device.

Impedance control and admittance control are dual not only in their cause-and-effect structure, but also in their performance. The impedance controlled device is typically lightweight, backlash free, and renders low mass [5]. Consequently, performance is lacking in the region of higher forces, high mass, and high stiffness. Adding complex end effectors is also a problem. Admittance controlled interfaces on the other hand, are capable of rendering very high stiffnesses and minimal friction. However, in order to prevent the device from becoming unstable, a small mass needs to be rendered constantly. Admittance controlled devices are very suitable for larger workspaces and for carrying complex end effectors with many degrees of freedom. Moreover, because forces are sensed rather than computed in real time, admittance control has the advantage of reduced modeling computation load.

The haptic interface selected to develop the Virtual Gearshift Application is a commercially available admittance controlled device [6] (Figure 2). The reason for this choice is that in order to simulate the gearshift feel of a manually operated transmission, it is expected that high force and high stiffness need to be rendered, which an impedance device is not capable of. Moreover, during gear shifting, the operator performs quick movements, which result in great accelerations of the end effector, for which an admittance controlled interface offers a more accurate and more stable simulation.

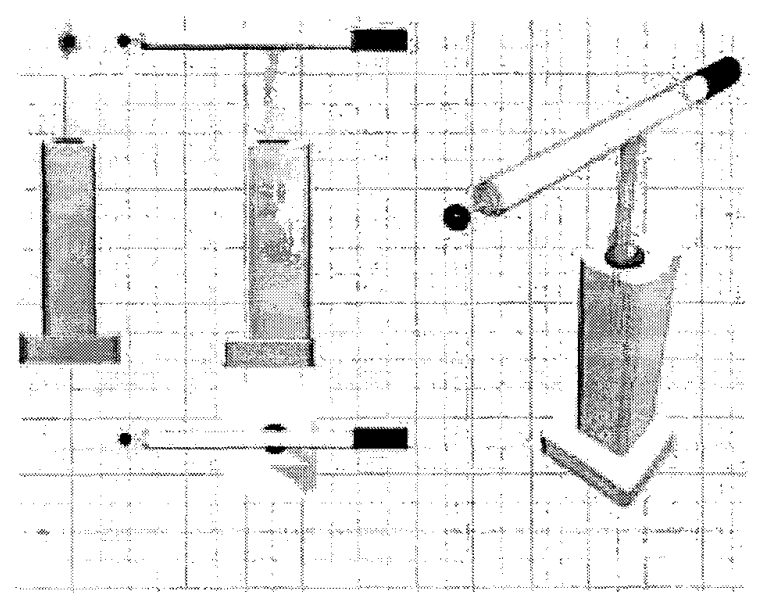

Fig. 2. The haptic interface selected for the Virtual Gearshift Application.

\section{Design of the Virtual Environment}

The interaction between operator and gear knob is simulated by programming the haptic device. Therefore, out of the measurement results, a mechanical model of the gearbox is extracted. It only contains those parts that mainly cause the forces induced on the operator's hand. These are the locking mechanism and the synchronization unit of the gearbox, which are modeled as compliance and Coulomb friction respectively.

Several iterations in design are made by measuring the interaction at the end effector of the haptic device and comparing these measurements to the interaction measured in the test vehicle. This way the mechanical model is finetuned so that the measured simulated interaction resembles the measured real interaction as closely as possible.

In order to provide a true illusion of gear shifting, not only the interaction between operator and gear knob should be simulated, but this interaction should also be embedded in a realistic context. That is why the haptic device is placed inside a mock-up of the test vehicle, shown in Figure 3. This is done in such a way that the location of its end effector exactly coincides with the location the gear knob used to be.

In order to evaluate gearshift feel, influence of visual differences between the real gearlever and the haptic device on the appreciation of the virtual gearbox should be avoided. In normal driving situations drivers do not look at the gearlever while changing gears either. To distract drivers' visual attention from the gearlever in order to force drivers to change gears based on feel, messages are shown on a big screen in front of the mock-up. 

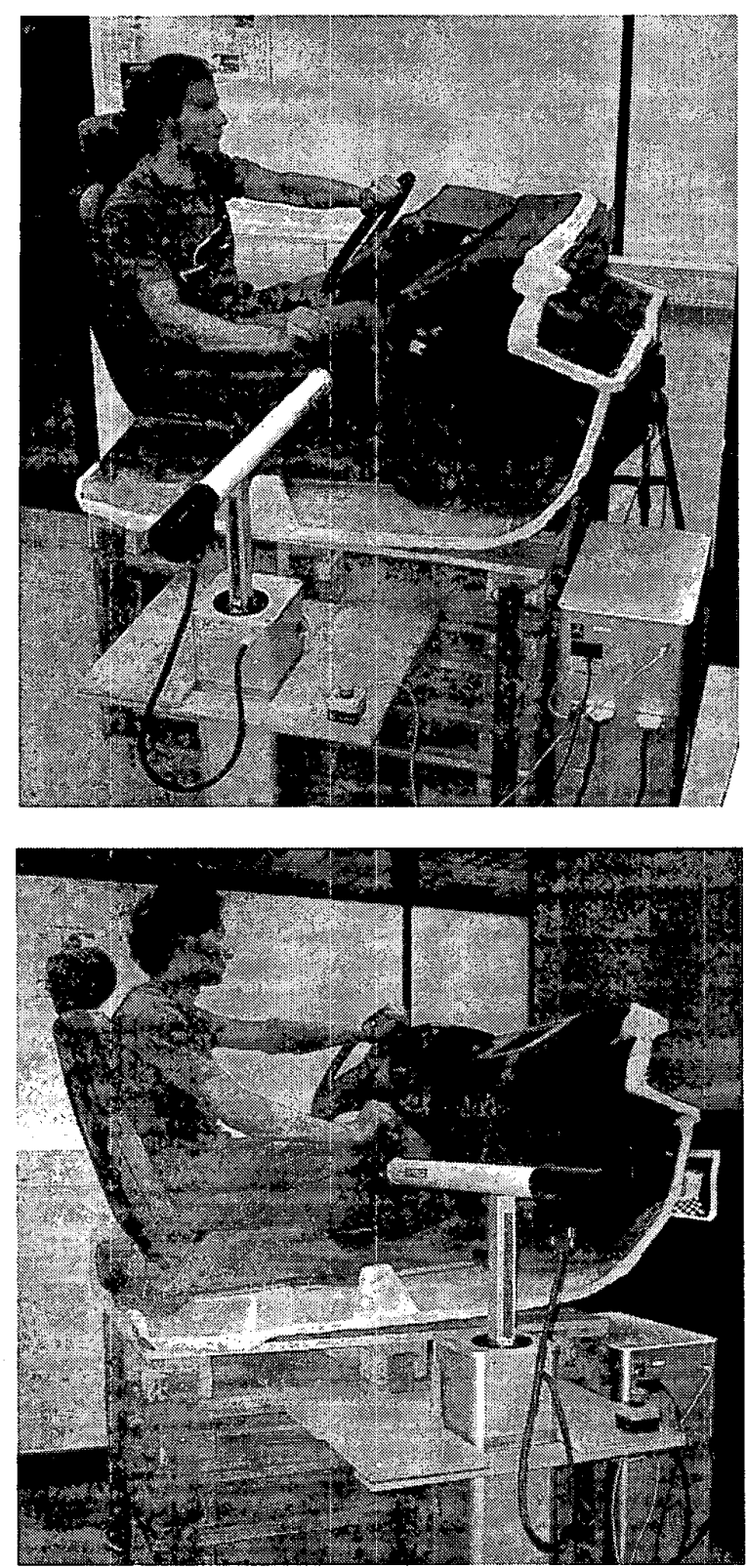

Fig. 3. The Virtual Gearshift Application.

\section{Evaluation of the Virtual Gearshift APPLICATION}

\section{A. Evaluation Setup}

The objective of the evaluation is to determine the quality of the designed Virtual Gearshift Application. As the quality of a virtual environment is determined by the degree to which the user gets the same experience as he would have had in the real world, the evaluation should consist of comparing the virtual gear shifting experience to the real experience. Ideally, this would be done by blindfolding a participant and placing him inside of the driver's seat of either the test vehicle or the Virtual Gearshift Application. If participants would not be able to discriminate between the gearshift feel of the real and the virtual gearbox, the quality of the simulation would be optimal. Unfortunately, due to the fact that other clues (e.g. the way of entrance to the mock-up and the test vehicle respectively) could play a part in discrimination between both "gearboxes", this method can not be used. Therefore, the user experiences are measured and compared by exploiting a questionnaire. A completed questionnaire should give a personal opinion on the gearshift feel. By comparing a participant's opinion of the real and the virtual gearbox, the quality of the application can be determined.

\section{B. Participants}

Ten participants are selected according to a number of criteria. First of all, participants have to possess a car with a manually operated transmission in which they have a significant amount of driving experience. This way, they have a solid reference while judging the gearshift feel of the test vehicle and the Virtual Gearshift Application respectively. Additionally, participants should represent the age group containing most new car buyers. However, no participants over 50 are selected since above the age of 50 physical and cognitive functions begin to degenerate. Therefore, participants needed to be aged between 20 and 50. Finally, in order to exclude the effect of gender, the number of male participants needed to equal the number of female participants.

\section{Evaluation Method}

The questionnaire comprises four types of questions. Part A contains some general questions that should capture the gearbox's general gearshift feel. Participants indicate to what degree the gearshift feel complies with two extremities. A five point scale is used, because this offers sufficient opportunity for differentiation. Since humans are not very sensitive in the "haptic domain" (i.e. humans have difficulty to discriminate between two forces, compliances, viscosities or similar quantities that only differ slightly) it is expected that using a scale with more opportunity for differentiation would not offer more accuracy. It is also expected that participants may take their own car as a reference while answering the questions. An example of this type of question is:

How much effort does it take to operate the gearbox? little $1 \begin{array}{llllll}1 & 2 & 3 & 4 & 5 & \text { much }\end{array}$

In part $B$, the gearshift feel of the individual forward gears can be judged. Instead of giving an indication on a scale, participants are asked to select the gear to which the 
question applies most. Selection of "no difference" also is an option. An example of this type of question is:

Which gear engages least easy?

$1 / 2 / 3 / 4 / 5 /$ no difference

As the mechanical construction of the reverse gear is fundamentally different from that of the forward gears, part $\mathrm{C}$ of the questionnaire deals with the reverse gear compared to the forward gears. An example of this type of question is:

Compared to the forward gears, how much effort does it take to engage the reverse gear? more / less / no difference

Finally, in part D, the neutral position of the gearbox can be evaluated. Without engaging a gear, the participant is asked to compare movements to the right and to the left of neutral position. An example of this type of question is:

What takes more effort, a quick move to the right or a quick move to the left of neutral position? left / right / no difference

For every participant, it is determined to what degree the response on the real gearbox is similar to that on the Virtual Gearshift Application. Therefore, the completed questionnaire after shifting in the test vehicle is compared to the one after shifting in the Virtual Gearshift Application. The higher the degree of similarity on a particular question, the better the test vehicle's gearbox is simulated by the Virtual Gearshift Application.

\section{Evaluation Results}

After evaluation of the scores, it turned out that participants often gave a similar (part A) or equal (parts B, $\mathrm{C}$ and $\mathrm{D})$ judgment. Apart from some minor discrepancies that should be corrected in a future redesign, generally, the simulated gearshift feel was judged to be similar to the original gearshift feel. The most convincing indication that the Virtual Gearshift Application simulates the gearshift in a natural way is the observation that during the usability testing, participants were able to look upon the Virtual Gearshift Application as if it were a real gearbox: they treated it the way a gearbox is naturally treated and they spoke about it as if it were real, e.g. "I feel more play than in my own car" or "Switching form 3rd to 4th gear goes smoother than from 1 st to 2 nd gear". It is therefore concluded that it indeed is possible to create an application that realistically simulates the gearshift feel of a manual transmission in a passenger car.
The two main discrepancies found between the real and the virtual gearbox are:

1) Operation of the Virtual Gearshift Application requires more effort than operation of the test vehicle's gearbox;

2) The gearlever of the Virtual Gearshift Application feels stiffer than the gearlever of the test vehicle.

These findings should be the input for a future redesign of the Virtual Gearshift Application.

Further analysis of these outcomes revealed that:

1) The effort required for engagement of a gear is judged on the basis of the total work (force times displacement) performed rather than on the basis of maximal required forces;

2) While operating a transmission in a passenger car, actions far from the body are judged to require more effort than actions close to the body.

\section{CONCLUSION}

Apart from some minor discrepancies that should be corrected in a future redesign, generally, the simulated gearshift feel was judged to be similar to the original gearshift feel. Moreover, during the usability testing, participants were able to look upon the Virtual Gearshift Application as if it were a real gearbox. So, the final conclusion is that it indeed is possible to create an application that realistically simulates the gearshift feel of a manual transmission in a passenger car.

The present design of the Virtual Gearshift Application however only simulates gearshift feel under "showroomshifting" circumstances, i.e. while the vehicle is at rest, the engine is not running, and the clutch is disengaged. In order to provide a simulation of the gearshift feel under driving conditions, the mechanical model of the gearbox needs to be enhanced. It should then become possible to define the desired gearshift feel, after which the physical gearbox is designed in such a way that it matches the desires. In this way, the customer will be able to specify the product behavior in a direct and clear way. As a result, gearboxes can be designed or adjusted for groups of drivers (old/young, sporty/comfy).

Besides adding dynamic characteristics to the gearshift feel, other components could be added to the designed Virtual Prototyping Environment as well, e.g. haptics for simulation of the clutch feel, the steering feel or the suspension feel. The Virtual Gearshift Application then grows from a VPE for gear shifting only to a VPE for a complete experience of driving a passenger car. This way, all kinds of personal preferences with respect to driving behavior could be identified and as a next step be physically realized. 


\section{REFERENCES}

[1] F. J. A. M. van Houten, "The Use and Development of Haptic Devices and Virtual Reality as Engineering Tools", Proceedings of the 34th CIRP International Seminar on manufacturing systems, pp. 275-283, 2001.

[2] R. J. Adams, Stable Haptic Interaction with Virtual Environments, University of Washington, 1999.

[3] J. Salisbury \& M. Srinivasan, Virtual Environment Technology for Training (VETT), 1992. In: G. Burdea, Force and Touch Feedback for Virtual Reality, John Wiley \& Sons, 1996, ISBN 0-471-02141-5.

[4] G. Burdea, Force and Touch Feedback for Virtual Reality, John Wiley \& Sons, 1996, ISBN 0-471-02141-5.

[5] R. J. Adams \& B. Hannaford, Control Law Design for Haptic Interfaces to Virtual Reality, 2001. In: R. Q. van der Linde, P. Lammertse, E. Frederiksen, \& B. Ruiter, "The HapticMaster: a New High-Performance Haptic Interface", Proceedings of Eurohaptics, pp. 1-5, 2002.

[6] R. Q. van der Linde, P. Lammertse, E. Frederiksen, \& B. Ruiter, "The HapticMaster: a New High-Performance Haptic Interface", Proceedings of Eurohaptics, pp. 1-5, 2002. 